\title{
Progressões geométricas e o estudo da matemática financeira
}

\author{
Márcio Rodrigues Vitor Petry
}

\begin{abstract}
Resumo
Neste trabalho são apresentadas atividades desenvolvidas com alunos do Ensino Médio, buscando mostrar a relação entre problemas de matemática financeira e progressão geométrica (PG). As atividades desenvolvidas e as discussões estabelecidas permitiram aos alunos a compreensão de conceitos relacionados à matemática financeira através de uma sequência numérica, de forma a proporcionar um aprendizado baseado na construção do conhecimento, onde situações do dia a dia são discutidas, facilitando a tomada de decisões que podem influenciar a vida financeira dos cidadãos. Proporcionou-se assim o processo de ensino e aprendizagem tendo o aluno como protagonista de sua formação.
\end{abstract}

Palavras-chave: Progressão geométrica; Matemática financeira; Aprendizagem; Sistema de amortização francês.

\section{Abstract}

In this work are presented activities developed with high school students, trying to show the relationship between problems of financial mathematics and geometric progression (GP). The activities developed and the discussions established allowed the students to understand concepts related to financial mathematics through a numerical sequence, in order to provide learning based on the construction of knowledge, where everyday situations are discussed, facilitating the decisions that can influence the financial life of citizens. The teaching and learning process was thus provided with the student as the protagonist of his/her training.

Keywords: Geometric progression; Financial math; Learning; French amortization system.

\section{Introdução}

Diante das estatísticas sobre a avaliação do ensino de matemática no Brasil fica evidente a necessidade de se promover melhorias que passam pela realização de trabalhos diferenciados na forma de ensinar Matemática, despertando o interesse e a motivação dos alunos para facilitar o processo de aprendizagem. Neste sentido, destaca-se a necessidade de apresentar os conteúdos e conceitos matemáticos de forma que possam ser compreendidos e tenham significado para os alunos, além da consideração de seus conhecimentos prévios em relação aos conceitos a serem trabalhados. De acordo com [1], autor que propôs o conceito da aprendizagem significativa, o fator mais 
importante que influencia o aprendizado é aquilo que o aprendiz já conhece [1]. Para conhecermos as percepções prévias dos alunos sobre tópicos de matemática financeira, estes responderam a um questionário elaborado para esta finalidade. Além disso o primeiro autor era o professor de matemática responsável pela a turma que participou do desenvolvimento das atividades, o que contribuiu para o conhecimento e a compreensão destas percepções.

O estudo da matemática financeira possibilita a compreensão de conceitos matemáticos de forma simples e de aplicação visível para o aluno, onde é possível perceber que todos podem aprender conceitos básicos de matemática que contribuirão para a melhoria de sua qualidade de vida. Esta percepção pode servir de elemento motivador para o trabalho de conceitos matemáticos em sala de aula. Neste sentido é fundamental que as aulas sobre matemática financeira não se restrinjam apenas a conceitos de juros simples e compostos, sem a devida contextualização que gere a compreensão de significados. Consideramos ser viável estabelecer relações entre os conceitos relacionados à matemática financeira e ao estudo de progressões, possibilitando conexões desses tópicos com situações do cotidiano dos alunos.

Segundo Ausubel [1], quando as informações são aprendidas sem interagir com conceitos relevantes na estrutura cognitiva, temos uma aprendizagem mecânica. Para compreender a relevância dos conceitos estudados, os sujeitos envolvidos no estudo fizeram consultas em estabelecimentos comerciais, concessionárias de veículos, bancos e financeiras com a finalidade de observar as principais formas de financiamento adotadas pelo mercado, chegando a conclusão de que o Sistema de Amortização Francês (SAF) é muito utilizado em financiamentos. O presente trabalho é resultado das atividades desenvolvidas em uma dissertação de mestrado do PROFMAT [6] onde trabalhou-se com alunos do Ensino Médio com a finalidade de estabelecer relações entre os conceitos envolvendo o estudo de progressões geométricas (PG) e o SAF, de forma a explicitar os significados desses conceitos para favorecer o processo de ensino e aprendizagem. Para Ausubel aprender significativamente é ampliar e reconfigurar ideias já existentes na estrutura mental e com isso ser capaz de relacionar novos conteúdos ([3]). Entende-se que esta forma de trabalho permite aos alunos o exercício de sua cidadania através de atitudes positivas, como a autonomia, confiança quanto as capacidades matemáticas e perserverança na solução de problemas, despertando o interesse para um assunto que gera muitas dúvidas e discussões na sociedade. Também observou-se o desenvolvimento de algumas habilidades de cálculo e a compreensão do significado e da demonstração de propriedades e teoremas relativos ao conteúdo de PG e a conceitos de matemática financeira. Dessa forma, o aprendizado deixou de ser um processo passivo, visto que este contribuiu para que o educando desenvolvesse potencialidades e a capacidade de pensar na tomada de decisões a partir dos conceitos aprendidos.

O objetivo deste trabalho é de apresentar algumas atividades desenvolvidas com alunos do Ensino Médio que mostram a possibilidade de trabalhar de forma integrada conceitos de progressões e de matemática financeira. Inicialmente foi aplicado um questionário para a identificação dos conhecimentos prévios dos sujeitos da pesquisa referente ao tema em discussão. A partir da avaliação de suas respostas, foram planejadas atividades de intervenção que culminaram no desenvolvimento do projeto. A aprendizagem foi observada de forma contínua ao longo de todo o desenvolvimento das atividades e através da aplicação de um novo questionário (pós teste) onde os alunos responderam questões sobre os tópicos desenvolvidos.

Apresentou-se aos alunos uma relação de exemplos de problemas de matemática financeira envolvendo situações com as quais muitos deles se deparam com certa frequência. Alguns dos exemplos foram elaborados pelos autores e outros obtidos em livros didáticos. A finalidade da apresentação 
desses exemplos foi a de instigar os alunos para solucionar tais problemas e em não conseguindo resolvê-los com os conceitos matemáticos já conhecidos por eles, motivá-los para a aprendizagem de novos conceitos a fim de resolver os problemas em questão. Alguns dos problemas propostos aos alunos são apresentados ao longo do texto com o intuito de analisar o processo de aprendizagem ocorrido durante o desenvolvimento das atividades.

As atividades a que se refere o presente trabalho foram desenvolvidas junto a alunos do Ensino Médio de uma escola pública no interior do estado de São Paulo. Na sequência, apresenta-se um breve comentário sobre os juros compostos e equivalência de capitais acompanhados de algumas das discussões feitas com os alunos a cerca da resolução de exemplos envolvendo este estudo. O SAF também é apresentado, abordando conceituação, sua origem e utilização, acompanhado da análise de alguns exemplos de aplicações trabalhadas em sala de aula, buscando identificar o significado das expressões envolvidas. Apresentam-se aplicações do SAF em situações reais, onde é oportunizada aos alunos a compreensão dos conceitos através da representação de dados em planilhas eletrônicas. Também são apresentadas atividades que relacionam o estudo de PG com a matemática financeira, utilizando resultados de PG para resolver problemas do SAF. Por fim, fazse uma reflexão dos resultados observados durante a aplicação e o desenvolvimento das atividades junto aos alunos.

\section{Juros compostos}

No sistema de juros compostos, deve-se calcular o juros no fim de cada período, formando um montante sobre o qual se calculam os juros do período seguinte, até esgotar o tempo de aplicação ([2]). Para motivar a introdução desse tema, foram trabalhados problemas de juros compostos, associando a sua resolução ao desenvolvimento de conceitos de PG. Para a percepção da necessidade de estabelecer uma relação geral, foi proposto aos alunos o problema inverso dado pelo exemplo 1 .

Exemplo 1. Qual deve ser o tempo de integralização para que a quantia de $R \$ 30.000,00$ gere um montante de $R \$ 32.781,81$, quando aplicada a uma taxa de $3 \%$ ao mês, no sistema de juros compostos?

A partir dos problemas desenvolvidos anteriormente usando indução matemática, chegou-se à expressão para o cálculo do juro composto que é enunciado no teorema que segue ([4, p45]), cuja demonstração não será apresentada neste trabalho, porém foi realizada com os alunos.

Teorema 1. No regime de juros compostos de taxa $i$, um principal $M_{o}$ transforma-se, em $n$ periodos de tempo, em um montante igual a:

$$
M_{n}=M_{o} \cdot(1+i)^{n} .
$$

Usando a expressão (1) e as propriedades de logaritmos, que já haviam sido discutidas anteriormente com os alunos, o problema proposto foi resolvido considerando que $M_{o}=\mathrm{R} \$ 30.000,00, i=$ $3 \%$ ao mês, $M=\mathrm{R} \$ 32.781,81$ e $n$ é a incógnita:

Temos, $M_{n}=M_{o} \cdot(1+i)^{n},(1+i)^{n}=\frac{M_{n}}{M_{o}},(1,03)^{n}=\frac{32781,81}{30000}=1,092727$, assim,

$$
n=\frac{\log (1,092727)}{\log (1,03)}=3 \text {. }
$$


Portanto o tempo deve ser de 3 meses.

A partir desse desenvolvimento, vários outros problemas foram propostos e resolvidos pelos alunos.

\section{Equivalência de capitais}

Sabe-se que o valor de uma quantia depende da época a qual ela está referida. Veja que, em uma situação onde o dinheiro rende $1 \%$ ao mês, pagar $\mathrm{R} \$ 100,00$ hoje ou pagar $\mathrm{R} \$ 101,00$ daqui a um mês é equivalente.

Discutindo algumas situações com os alunos de Ensino Médio e propondo alguns exemplos percebeu-se algumas dificuldades na compreensão quanto ao fator deslocamento no tempo, evidenciando-se a necessidade de fazer algumas exposições sobre os conceitos importantes envolvidos nestas situações. Essas discussões se deram a partir dos exemplos que seguem. O exemplo dois foi adaptado de [4, p46].

Exemplo 2. Um indivíduo tomou um empréstimo de $R \$ 500,00$ a juros mensais de $10 \%$. Dois meses após, este indivíduo pagou $R \$ 250,00$ e no mês seguinte liquidou o seu débito. Qual o valor desse último pagamento?

Realizou-se a análise do problema proposto com os alunos, conforme descrição que segue: de acordo com o Teorema $1, M_{n}=M_{o} \cdot(1+i)^{n}$ é uma quantia relacionada ao tempo atual, ou seja, $M_{o}$ transformar-se-á, após $n$ períodos de tempo em $M_{o} \cdot(1+i)^{n}$, isto é, uma quantia, cujo valor atual é $M_{o}$ equivale no futuro, depois de $n$ períodos de tempo a $M_{n}=M_{o} \cdot(1+i)^{n}$. Daí pode-se concluir que para descobrir o valor futuro deve-se multiplicar por $(1+i)^{n}$. Reciprocamente, para descobrir o valor atual, deve-se dividir por $(1+i)^{n}$.

A partir dessa análise a resolução do problema fica da seguinte forma:

Considerando inicialmente que o valor atual do empréstimo é de $\mathrm{R} \$ 500,00$ equivale a um valor futuro

$$
500=\frac{250}{(1+0,1)^{2}}+\frac{P}{(1+0,1)^{3}} .
$$

visto que foi feito um pagamento de $\mathrm{R} \$ 250,00$ após dois meses e um pagamento $P$ após três meses. Segue então que $P=\mathrm{R} \$ 390,50$.

Outra situação que levou a várias discussões em aula foi o problema proposto no exemplo 3, adaptado de [4, p47]:

Exemplo 3. Um produto é oferecido em uma loja nas seguintes condições:

a) $\grave{A}$ vista, com $10 \%$ de desconto.

b) Em duas prestações mensais iguais, sem desconto, vencendo a primeira um mês após a compra.

c) Em três prestações mensais iguais, sem desconto, vencendo a primeira no ato da compra.

Qual a melhor opção para a compra desse produto, se o dinheiro vale, para o comprador, $5 \%$ ao mês?

Para facilitar a discussão, fixou-se, sem perda de generalidade, um preço de $\mathrm{R} \$ 30,00$. Para pagamento à vista rapidamente os alunos concluíram que o valor seria de $\mathrm{R} \$ 27,00$. Assim, a discussão ficou em torno dos itens b e c, cujo resultado segue, comparando em cada uma das situações os valores pagos futuramente com os valores atuais, considerando uma atualização monetária de $5 \%$ ao mês, conforme previsto no exemplo: 
Analisando o item b, tem-se: $V_{b}=\frac{15}{1,05}+\frac{15}{1,05^{2}} \approx 27,89$.

Analisando o item c, tem-se: $V_{c}=10+\frac{10}{1,05}+\frac{10}{1,05^{2}} \approx 28,59$.

Após cada uma dessas análises, amplamente discutidas e justificadas com os alunos, conclui-se que a melhor opção é a compra à vista. Destaca-se neste caso a importância de não considerar apenas as taxas praticadas pelo mercado, mas também, o rendimento que o consumidor eventualmente pode ter ao ficar com seu dinheiro por mais tempo, no caso de uma compra à prazo. Novamente aproveitou-se o exemplo para ampliar a discussão sobre equivalência de capitais. Outras situações problemas foram analisados com os alunos envolvendo a aplicação do Teorema 1 e suas consequências. Várias situações são encontradas em [4].

Dando sequência ao trabalho, foi introduzido o conceito de séries de pagamentos uniformes, cuja ilustração é feita com o exemplo 4, adaptado de [4, p51].

Exemplo 4. Um produto cujo preço à vista era de $R \$ 1.000,00$ foi comprado em 6 prestações iguais, sendo a primeira paga um mês após a compra. Se os juros são de $10 \%$ ao mês, determine $o$ valor das prestações.

Usando as idéias discutidas anteriormente, estabeleceu-se a expressão que fornecesse o valor atual de cada parcela futura, de forma que a soma de todas as parcelas representasse o valor à vista do produto, conforme segue:

$$
1000=\frac{P}{1,1}+\frac{P}{1,1^{2}}+\frac{P}{1,1^{3}}+\frac{P}{1,1^{4}}+\frac{P}{1,1^{5}}+\frac{P}{1,1^{6}}
$$

Dessa forma, o problema ficou caracterizado como uma soma dos termos de uma PG em que seu primeiro termo é $a_{1}=\frac{P}{1,1}$ e sua razão é $q=\frac{1}{1,1}$. Usando os conhecimentos relativos a soma dos termos de uma PG finita, foi possível chegar ao valor de cada prestação, conforme segue:

$$
1.000=\frac{a_{1}\left(q^{n}-1\right)}{q-1}=\frac{\frac{P}{1,1}\left[\left(\frac{1}{1,1}\right)^{6}-1\right]}{\frac{1}{1,1}-1}
$$

de forma que $P=\mathrm{R} \$ 229,60$.

Acredita-se que estas discussões são fundamentais para que os alunos não fiquem apenas fazendo contas e substituições de valores em fórmulas prontas sem estabelecer significados para o que estão fazendo, sem a devida compreensão. Estabeleceu-se na análise desse exemplo uma estreita relação entre o ensino de progressões geométricas e a compreensão de conceitos e de resultados de matemática financeira, de fundamental importância para a administração financeira de qualquer cidadão.

Após a compreensão do processo, foi proposto uma generalização para o cálculo do valor da parcela em séries de pagamentos uniformes, cujo resultado segue enunciado no Teorema 2, disponível em [4] que foi demonstrado, usando o mesmo raciocínio proposto no exemplo 4, porém de forma algébrica. 
Teorema 2. O valor de uma série uniforme de $n$ pagamentos iguais a $P$, uma unidade de tempo antes do primeiro pagamento, é

$$
P_{v}=P\left[\frac{1-(1+i)^{-n}}{i}\right]
$$

sendo $i$ a taxa de juros e $P_{v}$ pagamento à vista.

Demonstração. Seguindo os argumentos utilizados no exemplo anterior, escreve-se o valor do pagamento à vista como uma soma de parcelas futuras em valores atuais, conforme segue:

$$
P_{v}=\frac{P}{(1+i)}+\frac{P}{(1+i)^{2}}+\cdots+\frac{P}{(1+i)^{n}}
$$

utilizando a soma dos termos de uma PG finita obtém-se:

$$
\frac{P_{v}}{P}=(1+i)^{-1} \cdot \frac{(1+i)^{-n}-1}{(1+i)^{-1}-1}=\frac{(1+i)^{-n}-1}{(1+i) \cdot\left(\frac{1}{1+i}-1\right)}=\frac{(1+i)^{-n}-1}{\frac{(1+i) \cdot(1-1-i)}{1+i}}=\frac{(1+i)^{-n}-1}{-i}
$$

de onde segue que:

$$
P_{v}=P \cdot\left[\frac{1-(1+i)^{-n}}{i}\right]
$$

Após a generalização, vários problemas foram propostos e resolvidos pelos alunos. Para dar continuidade ao trabalho com os alunos, foi solicitado que estes realizassem uma pesquisa sobre os principais modelos usados para financiamentos de bens como veículos e imóveis. Nesta pesquisa, destacaram-se dois sistemas de amortização que foram estudados em aula: O SAF e o SAC (sistema de amortização constante). O estudo do SAF será abordado na sequência desse trabalho.

\section{Sistema de Amortização Francês (SAF)}

O SAF, também conhecido como tabela Price, é muito utilizado nos cálculos de financiamentos ([4]). Segundo [5], a denominação tabela Price é em homenagem ao filósofo, teólogo e matemático inglês Richard Price, que viveu no século XVIII e incorporou a teoria dos juros compostos às amortizações de empréstimos. O nome francês deve-se ao fato do sistema ser desenvolvido na França, no século XIX.

De acordo com [7, p220], "esse sistema consiste em um plano de amortização de uma dívida em prestações periódicas iguais e sucessivas, dentro do conceito de termos vencidos, em que o valor de cada prestação, ou pagamento, é composto por duas parcelas distintas: uma de juros e outra de capital (chamada amortização)."

Apesar da pesquisa feita pelos alunos apontar este sistema como um dos mais usados pelo mercado, principalmente em financiamentos de curto e médio prazo, como é o caso do financiamento de automóveis, observou-se que a maioria dos alunos tiveram dificuldade de compreender o funcionamento desse sistema. Foi necessário explorar os conceitos trazidos por eles a partir de exemplos para a 
adequada compreensão do sistema de amortização em questão. Uma das percepções apresentadas pelos alunos a partir da pesquisa foi a de que se tratava de um sistema de amortização em que o valor das parcelas era constante. Dessa forma a discussão passou a ser vinculada ao estudo anterior, encarando o problema como uma série de pagamentos uniformes. Os alunos trouxeram para a aula vários exemplos e tabelas em planilhas já prontas que serviram de base para o início dos estudos. O exemplo 5, adaptado de [4, p52], serviu para a construção de uma tabela Price com os alunos utilizando uma planilha eletrônica, buscando compreender o significado dos dados em uma tabela Price.

Exemplo 5. Uma pessoa deseja fazer um empréstimo de $\mathrm{R} \$ 1.000,00$ e pagar este empréstimo em 6 parcelas iguais, a uma taxa de $5 \%$ ao mês. Construa uma tabela para representar os dados utilizando o SAF.

Inicialmente foi calculado o valor de cada parcela, usando a equação (2), visto que o SAF é composto por parcelas de valor constante.

$$
1000=P \cdot\left(\frac{1-(1+0,05)^{-6}}{0,05}\right) \Rightarrow 50=P \cdot(0,253785) \Rightarrow P=\mathrm{R} \$ 197,02 .
$$

Assim, as seis parcelas são no valor de $\mathrm{R} \$ 197,02$, o que permitiu o início da construção da planilha, conforme mostrado na segunda coluna da tabela 1.

Note que $5 \%$ da dívida inicial que é de $\mathrm{R} \$ 1.000,00$ corresponde ao juro do primeiro mês, isto é, $J_{1}=\mathrm{R} \$ 50,00$. Assim, a amortização do primeiro mês $A_{1}$ é o valor da parcela subtraído do juro, ou seja, $A_{1}=\mathrm{R} \$ 197,02-\mathrm{R} \$ 50,00=\mathrm{R} \$ 147,02$. Dessa forma a dívida após o pagamento da primeira parcela (isto é, após o primeiro mês) passa a ser, $D_{1}=\mathrm{R} \$ 1000,00-\mathrm{R} \$ 147,02=$ $\mathrm{R} \$ 852,98$, conforme mostrado na linha correspondente ao primeiro mês na tabela 1 . De forma análoga, calculando os $5 \%$ dessa dívida tem-se um juros de $J_{2}=\mathrm{R} \$ 42,65$ e uma amortização de $A_{2}=\mathrm{R} \$ 197,02-\mathrm{R} \$ 42,65=\mathrm{R} \$ 154,37$. Portanto a dívida para o próximo mês é de $D_{2}=$ $\mathrm{R} \$ 852,98-\mathrm{R} \$ 154,37=\mathrm{R} \$ 698,61$ e assim sucessivamente até o pagamento da 6 parcela, quando a dívida é liquidada.

Tabela 1: Liquidação de dívida em tabela Price.

\begin{tabular}{|c|c|c|c|c|}
\hline Tempo $(n)$ & Parcelas $\left(P_{n}\right)$ & Juros $\left(J_{n}\right)$ & Amortização $\left(A_{n}\right)$ & Dívida $\left(D_{n}\right)$ \\
\hline 0 & - & - & - & $\mathrm{R} \$ 1000,00$ \\
\hline 1 & $\mathrm{R} \$ 197,02$ & $\mathrm{R} \$ 50,00$ & $\mathrm{R} \$$ & $\mathrm{R} \$ 852,98$ \\
\hline 2 & $\mathrm{R} \$ 197,02$ & $\mathrm{R} \$ 42,65$ & $\mathrm{R} \$ 154,37$ & $\mathrm{R} \$ 698,61$ \\
\hline 3 & $\mathrm{R} \$ 197,02$ & $\mathrm{R} \$ 34,93$ & $\mathrm{R} \$ 162,09$ & $\mathrm{R} \$ 536,52$ \\
\hline 4 & $\mathrm{R} \$ 197,02$ & $\mathrm{R} \$ 26,83$ & $\mathrm{R} \$ 170,19$ & $\mathrm{R} \$ 366,33$ \\
\hline 5 & $\mathrm{R} \$ 197,02$ & $\mathrm{R} \$ 18,31$ & $\mathrm{R} \$ 178,70$ & $\mathrm{R} \$ 187,63$ \\
\hline 6 & $\mathrm{R} \$ 197,02$ & $\mathrm{R} \$ 9,38$ & $\mathrm{R} \$ 187,63$ & Dívida liquidada \\
\hline
\end{tabular}

Utilizando planilha eletrônica foram criadas pelos alunos tabelas Price para a realização de simulações de vários problemas propostos em sala de aula, o que possibilitou a descoberta e discussão dos principais aspectos desse tipo de financiamento de forma que o estudo não se limitou a uma simples análise do comportamento dos juros compostos. Os resultados observados no desenvolvimento da tabela Price foram formalizados com os alunos através do teorema 3 , disponível em [4, p60]. 
Teorema 3. No sistema francês de amortização, sendo $i$ a taxa de juros e $n$ o número de pagamentos, temos:

$P_{k}=D_{o} \cdot\left(\frac{i}{1-(1+i)^{-n}}\right), D_{k}=D_{o} \cdot\left(\frac{1-(1+i)^{-(n-k)}}{1-(1+i)^{-n}}\right), J_{k}=i \cdot D_{k-1}, A_{k}=P_{k}-J_{k}$

sendo $P_{k}$ o valor da parcela, $D_{o}$ a divida inicial, $D_{k}$ o valor da dívida , $J_{k}$ o juro pago e $A_{K}$ a amortização, relativas à $k$-ésima parcela.

Observou-se ainda que ao lançar um número muito grande de parcelas, essa parcela passaria a não se alterar significativamente, concluindo-se que quanto menor o número de parcelas, menor é o valor total de juros a serem pagos. De fato, observou-se que no limite quando o número de parcelas tende ao infinito, o valor da parcela tende a um valor fixo que corresponde ao juro do primeiro mês. Essa situação foi discutida a partir do exemplo 6 .

Exemplo 6. Considere o financiamento de um automóvel pelo SAF com a primeira parcela sendo paga um mês após a compra e cujo valor financiado é de $R \$ 24.000,00$. Se a taxa de juros é de $2 \%$ ao mês, determine o valor das prestações para financiamento em 24, 60 e 120 meses. Faça uma simulação também para verificar o valor da parcela para uma situação hipotética de esse financiamento ser em 180, 360 e 720 meses. Faça uma análise do comportamento do valor da parcela para uma quantidade grande de parcelas.

Pelo desenvolvimento da soma dos termos da PG:

$$
24000=\frac{P}{1,02}+\frac{P}{1,02^{2}}+\frac{P}{1,02^{3}}+\cdots+\frac{P}{1,02^{24}}
$$

ou pela equação (2) segue que para pagamento da dívida em 24 meses, o valor de cada parcela fica $P=\mathrm{R} \$ 1.298,91$. De forma análoga, para as demais situações solicitadas no problema, obtém-se o valor da parcela, conforme ilustrado na tablela 2.

Tabela 2: Relação entre quantidade de parcelas e valor de cada parcela.

\begin{tabular}{|c|rc|}
\hline Número de parcelas & \multicolumn{2}{|c|}{ Valor da parcela } \\
\hline 24 & $\mathrm{R} \$ 1.298,91$ \\
\hline 60 & $\mathrm{R} \$$ & 690,43 \\
\hline 120 & $\mathrm{R} \$$ & 529,15 \\
\hline 180 & $\mathrm{R} \$$ & 493,99 \\
\hline 360 & $\mathrm{R} \$$ & 480,39 \\
\hline 720 & $\mathrm{R} \$$ & 480,00 \\
\hline
\end{tabular}

Após a simulação de vários financiamentos a longo prazo usando o SAF, os alunos perceberam que quando se aumenta indefinidamente o número de parcelas, a variação do valor das parcelas fica cada vez menor. Voltando ao problema proposto no exemplo 6, ao considerar mais de 720 parcelas, a variação ocorreria apenas a partir da quarta casa decimal, que para valores monetários é desprezada. Na prática, isto significa que se continuar aumentando o número de parcelas, o valor da parcela fica inalterado. Essa discussão motivou o estudo de progressões geométricas infinitas, para compreender o que ocorre nestas situações do ponto de vista matemático. 
Considerando uma PG de termo geral $a_{n}=a_{1} \cdots q^{n-1}$, a soma de seus termos é dada por:

$$
S_{n}=\frac{a_{1} \cdot\left(q^{n}-1\right)}{q-1}, q \neq 1
$$

Para $|q|<1$, a soma $S_{n}$ em (3) tem um limite finito quando $n \rightarrow \infty$, pois em

$$
\lim _{n \rightarrow \infty} q^{n}=0, \text { quando }|q|<1 \Rightarrow \lim _{n \rightarrow \infty} S_{n}=\frac{a_{1} \cdot(0-1)}{q-1}
$$

e portanto:

$$
\lim _{n \rightarrow \infty} S_{n}=\frac{a_{1}}{1-q}
$$

Quando $|q| \geq 1, q^{n}$ diverge e portanto $S_{n}=\frac{a_{1} \cdot\left(q^{n}-1\right)}{q-1}$ diverge quando $n \rightarrow \infty$ e essa soma representa uma $\mathrm{PG}$ divergente.

Embora não seja objetivo discutir formalmente conceitos de limite no Ensino Médio, a compreensão intuitiva é perfeitamente possível e a notação usada para sua representação também é compreendida pelos alunos. Observou-se com os alunos que nos casos estudados envolvendo situações de financiamento, a razão da PG é dada por $q=\frac{1}{1+i}$ de forma que $|q|<1$, visto que $i>0$. Assim, a soma converge. Voltando ao exemplo 6, usando a equação (4) tem-se:

$$
\begin{aligned}
& 24.000,00=\frac{P}{1,02}+\frac{P}{1,02^{2}}+\frac{P}{1,02^{3}}+\cdots=P \cdot\left(\frac{1}{1,02}+\frac{1}{1,02^{2}}+\frac{1}{1,02^{3}}+\cdots\right) \\
&=P \cdot\left(\frac{\frac{1}{1,02}}{1-\frac{1}{1,02}}\right)=P \cdot\left(\frac{\frac{1}{1,02}}{\frac{1,02-1}{1,02}}\right)=P \cdot\left(\frac{1}{0,02}\right)=50 P
\end{aligned}
$$

de forma que ao considerar "infinitas" parcelas, o valor de cada parcela fica: $P=\mathrm{R} \$ 480,00$.

Isto significa dizer que aumentando indefinidamente o número de prestações, o valor da parcela tende a um valor fixo não havendo possilidade de ficar abaixo desse valor. Observando o problema, os alunos perceberam que $2 \%$ do valor da dívida de $\mathrm{R} \$ 24.000,00$ corresponde a $\mathrm{R} \$ 480,00$. Isto significa que ao aumentar indefinidamente o número de prestações, o consumidor passa a pagar apenas o juro de sua dívida e não faz a amortização da mesma. Esse raciocínio é confirmado ao generalizar a situação discutida anteriormente no exemplo.

Consideramos uma dívida de valor atual $D_{0}$ a uma taxa de juros $i$ para pagamento em $n$ parcelas iguais e sucessivas, vencendo a primeira um período de tempo após o instante atual. Considerando que $P=P(n)$, se fizermos o número de parcelas crescer indefinidamente, isto é, fazendo $n \rightarrow \infty$, temos a soma de uma PG com infinitos termos. Segue então, usando a equação (4): 


$$
\begin{aligned}
D_{0} & =\lim _{n \rightarrow \infty}\left[\frac{P}{1+i}+\frac{P}{(1+i)^{2}}+\frac{P}{(1+i)^{3}}+\cdots+\frac{P}{(1+i)^{n}}\right] \\
& =\lim _{n \rightarrow \infty} P \cdot\left(\frac{1}{1+i}+\frac{1}{(1+i)^{2}}+\frac{1}{(1+i)^{3}}+\cdots\right) \\
& =\lim _{n \rightarrow \infty} P \cdot\left(\frac{\frac{1}{(1+i)}}{1-\frac{1}{(1+i)}}\right)=\lim _{n \rightarrow \infty} P \cdot\left(\frac{\frac{1}{(1+i)}}{\frac{(1+i)-1}{(1+i)}}\right)=\lim _{n \rightarrow \infty} P \cdot\left(\frac{1}{i}\right)
\end{aligned}
$$

ou seja: $\lim _{n \rightarrow \infty} P=i \cdot D_{0}$, o que significa que de fato o valor da parcela corresponde ao valor do juro mensal relativo à dívida, ou equivalentemente ao juro do primeiro mês, visto que a dívida se manterá constante. Note que $i \cdot D_{o}$ é o valor limite da prestação. Uma vez que no sistema monetário são usadas apenas duas casas decimais, pela definição de limite fica evidente que na prática poderá ser considerada a igualdade $P=i \cdot D_{0}$.

\section{Financiamento com carência para pagamento}

Em muitas situações práticas, o agente financeiro oferece uma carência para o início dos pagamentos, porém com a manutenção da incidência de juros sobre o valor da dívida. Ao tratar as questões de matemática financeira como aplicação do estudo da PG, esta situção fica de simples compreensão, conforme ilustrado no exemplo 7 , adaptado de [4, p63].

Exemplo 7. Um indivíduo fez uma compra no valor de $R \$ 2.000,00$ para pagamento em 12 parcelas mensais iguais a uma taxa de $2 \%$ ao mês. Qual o valor de cada parcela se a $1^{\text {a }}$ é paga:

(a) no ato da compra.

(b) cinco meses após a compra.

Para a solução do item (a), note que sobre a primeira parcela não poderá incidir juro, uma vez que ela é paga no ato da compra. Assim, o juro passa a ser contabilizado a partir da segunda parcela, conforme segue:

$$
\begin{aligned}
2000 & =P+\frac{P}{1,02}+\frac{P}{1,02^{2}}+\frac{P}{1,02^{3}}+\cdots+\frac{P}{1,02^{11}}=P \cdot\left(1+\frac{1}{1,02}+\frac{1}{1,02^{2}}+\frac{1}{1,02^{3}}+\cdots+\frac{1}{1,02^{11}}\right) \\
& =P \cdot \frac{\left[\left(\frac{1}{1,02}\right)^{12}-1\right]}{\frac{1}{1,02}-1}=P \cdot \frac{1,02^{-12}-1}{1,02^{-1}-1} \approx P \cdot(10,787)
\end{aligned}
$$

de onde segue, considerando arredondamento para duas casas decimais (sistema monetário), que: $P=185,41$.

Para a solução do item (b) devemos considerar que a primeira parcela será para 5 meses após a compra, de forma que seu valor já deve ser reajustado em 5 períodos. Assim, a expressão para a obtenção da parcela fica: 


$$
\begin{aligned}
2000 & =\frac{P}{1,02^{5}}+\frac{P}{1,02^{6}}+\frac{P}{1,02^{7}}+\cdots+\frac{P}{1,02^{16}}=\frac{P}{1,02^{5}} \cdot\left(1+\frac{1}{1,02}+\frac{1}{1,02^{2}}+\cdots+\frac{1}{1,02^{11}}\right) \\
& =\frac{P}{1,02^{5}} \cdot\left(\frac{\left[\left(\frac{1}{1,02}\right)^{12}-1\right]}{\frac{1}{1,02}-1}\right)=\frac{P}{1,02^{5}} \cdot\left(\frac{1,02^{-12}-1}{1,02^{-1}-1}\right) \approx P \cdot(9,77)
\end{aligned}
$$

o que resulta para o sistema monetário que: $P=204,71$.

Assim, o valor da prestação, para pagamento da primeira parcela após cinco meses é de R $\$ 208,31$.

\section{Análise do processo de aprendizagem}

Ao longo de todo processo de aplicação do projeto foram realizadas observações e anotações que possibilitaram avaliar e analisar o processo de aprendizagem dos conceitos discutidos com os sujeitos da pesquisa. Observou-se que ao trabalhar conceitos de matemática financeira como aplicação de outros conteúdos, em particular, da PG, o aluno consegue estabelecer com certa facilidade as relações entre o valor das parcelas e os períodos em que serão feitos os pagamentos, sem a necessidade de recorrer a muitas fórmulas prontas que em determinados momentos não fazem muito sentido para ele. Da forma como foi trabalhado o conteúdo proposto, os alunos mantiveramse motivados e participativos, pois ficou-lhes evidente a aplicabilidade dos conteúdos e tiveram consciência da importância desse conhecimento para seu cotidiano.

Com a orientação do professor, os alunos foram capazes de estabelecer associações entre os conteúdos trabalhados, percebendo a aplicação prática do estudo da PG em um contexto de muita importância para sua organização financeira. Observou-se intervenções e contribuições de forma a tornarem-se os protagonistas do processo de aprendizagem. Percebeu-se que a maioria dos alunos envolvidos neste trabalho conseguiu atribuir significados aos conceitos e às relações matemáticas trabalhadas.

Ao longo do trabalho desenvolvido, observou-se que além dos conceitos abordados de PG e de matemática financeira os alunos desenvolveram habilidades de trabalho com planilhas eletrônicas, organização de dados, análise dos sistemas financeiros e compreensão, mesmo que de forma bastante intuitiva e superficial, do conceito de limites.

A aplicação do questionário final mostrou que os sujeitos envolvidos ampliaram significativamente seus conhecimentos de matemática financeira e progressões, o que lhes permitiu desenvolver questões mais complexas sobre questões financeiras do cotidiano, conseguindo estabelçecer relações entre conceitos e mostrando capacidade de fazer planejamentos financeiros. Foi observada uma grande evolução dos alunos no período entre a aplicação dos dois questionários.

Entende-se que para ensinar e aprender matemática é necessário que sejam proporcionadas reflexões sobre o que e como trabalhar determinados conteúdos. É fundamental que o professor procure relacionar conteúdos que em muitas situações são trabalhados de forma desconexa, para facilitar a atribuição de significados ao que se ensina. Os alunos tiveram a percepção de que os conceitos matemáticos aprendidos não são independentes entre si. Espera-se que este trabalho contribua como uma sugestão para os docentes de matemática que queiram trabalhar os conteúdos de forma mais integrada. 


\section{Considerações finais}

No trabalho foram apresentadas atividades desenvolvidas com alunos do Ensino Médio, mostrando a possibilidade de trabalhar de forma integrada conceitos de diferentes conteúdos da Matemática. Nestas atividades foram trabalhados tópicos de matemática financeira integrados com conceitos de progressões geométricas. A avaliação das atividades feita pela observação dos sujeitos durante o desenvolvimento da pesquisa e dos questionários aplicados mostra que a estratégia se mostrou bastante eficiente, permitindo a ampliação significativa dos conhecimentos acerca dos temas discutidos.

\section{Agradecimentos}

O primeiro autor foi bolsista da CAPES pelo PROFMAT durante o desenvolvimento do projeto.

\section{Referências}

[1] Ausubel, D. P. A Aprendizagem Significativa: A Teoria de David Ausubel. São Paulo-SP: Moraes, 1982.

[2] Dante, L. R. Matemática: Contexto e Aplicações. São Paulo-SP: Editora Ática, 2011.

[3] Fernandes, E. David Ausubel e A aprendizagem Significativa. Revista Nova Escola, edição 248, 2011. Disponível em:

$<$ http://revistaescola.abril.com.br/formacao/david-ausubel-aprendizagem-significativa-662262. shtml>. Acesso em: 05 de julho de 2014.

[4] Morgado, A. C. Progressões e Matemática Financeira. Rio de Janeiro: SBM, Quinta Edição, 2010.

[5] Pereira, M. G. Plano Básico de Amortização pelo Sistema Francês e Respectivo Fator de Conversão. Dissertação de doutorado. São Paulo-SP: FCEA, 1965.

[6] Rodrigues, L. M. O Ensino da Matemática Financeira no Ensino Médio. Dissertação de mestrado, UTFPR-PROFMAT. Curitiba-PR, 2013.

[7] Sobrinho, J. D. V. Matemática Financeira. São Paulo-SP: Editora Atlas, Sétima Edição, 2000.

Márcio Rodrigues

E. E. Professor Pascoal Grecco e Colégio Lantagi - COC $<$ marluro2@yahoo.com.br>

Vitor Petry

Universidade Federal da Fronteira Sul - UFFS - Chapecó - SC $<$ vitor.petry@uffs.edu.br>

Recebido: 2015

Publicado: 2015 